\title{
A useful quantitative model for determination of enantiomeric composition of racemate praziquantel by ultraviolet spectroscopy combined with partial least squares and its application to praziquantel tablets
}

\author{
Man Zhao*, , Ran Meng*, ${ }^{*}$, Yifang Lu*, Lingyun $\mathrm{Hu}^{\dagger}, \mathrm{Na}$ Sun*, \\ Lei $\mathrm{Nie}^{*, *}, \|$ and Haina Wang, ${ }^{*}, \|$ \\ *School of Pharmaceutical Sciences, Shandong University \\ Jinan 250012, P. R. China \\ ${ }_{\dagger}^{\dagger}$ Shandong Cancer Hospital Affiliated to Shandong University \\ Jinan, P. R. China \\ *nielei2004@sdu.edu.cn \\ §whn2013@sdu.edu.cn
}

Received 16 August 2017

Accepted 12 December 2017

Published 18 January 2018

\begin{abstract}
A simple and novel method has been proposed to determine the enantiomeric composition of racemate praziquantel (PZQ) by using the analysis of ultraviolet (UV) spectroscopy combined with partial least squares (PLS). This method does not rely on the use of expensive carbohydrates such as cyclodextrins, but on the use of inexpensive sucrose, which is equally effective as carbohydrate. PZQ has two enantiomers. Through measuring the slight difference in the UV spectral absorption of $\mathrm{PZQ}$ due to different interactions between its two enantiomers and sucrose, the enantiomeric composition was determined by a quantitative model based on PLS analysis. The model showed that the correlation coefficients of calibration set and validation set were 0.9971 and 0.9972 , respectively. The root mean square error of calibration (RMSEC) and the root mean square error of prediction (RMSEP) were 0.0167 and 0.0129 , respectively. Then, the independent data of PZQ tablets were also used to test how well the quantitative model of PLS predicted the enantiomeric composition. The ratio of S-PZQ in tablet was 0.492 , determined by high-performance liquid chromatography as the reference value. Six solutions of the tablet samples were prepared, and the ratios of S-PZQ in tablet samples in the validation set were predicted by the PLS model. Their relative errors with the reference value were not more than $4 \%$. Therefore, the established model could be accurate and employed to predict the enantiomeric compositions of PZQ tablets.
\end{abstract}

Keywords: Enantiomeric compositions; praziquantel; partial least squares; ultraviolet spectroscopy; sucrose.

\footnotetext{
TThese authors contributed equally to the work and should be regarded as co-first authors.

$\|_{\text {Corresponding authors. }}$
}

This is an Open Access article published by World Scientific Publishing Company. It is distributed under the terms of the Creative Commons Attribution 4.0 (CC-BY) License. Further distribution of this work is permitted, provided the original work is properly cited. 


\section{Introduction}

Nowadays, more than one half of the drugs contain chiral centers, marketed not only as racemates but more and more as single enantiomer also. Two opposite enantiomers may exert different pharmacological properties, toxicities and side effects because of the stereoselective interaction with macromolecules in a biological system. ${ }^{1}$ For chiral drug, the structure-activity relationship is critically dependent on the degree of enantiopurity. During the production of a pharmaceutical product, the eudismic ratio, the potency ratio between the more active enantiomer (the eutomer) and the less active one (the distomer), should be taken into account. ${ }^{2}$ The Food and Drug Administration (FDA) requires, besides evaluating the effect of individual enantiomer, the determination of the purity of all chiral molecules produced, both at the industrial stage and in the final product. ${ }^{3}$ The determination of enantiomeric composition of pharmaceutical chiral product is of capital importance right now. ${ }^{4}$

Praziquantel (PZQ) is the world-recognized and most efficient drug for control and treatment of schistosomiasis before an effective vaccine comes out ${ }^{5-8}$ which is the drug recommended by World Health Organization (WHO) to treat schistosomiasis at present. ${ }^{9} \mathrm{PZQ}$ belongs to the pyrazine and isoquinoline compounds (shown in Fig. 1). Some works investigated the pharmacological action of PZQ according to its configurations and confirmed that the levoisomer (R-configuration) was the mainly effective component, whereas the S-enantiomer (S-PZQ) had no activity and was considered to be related to side effects. ${ }^{10-15}$ Additionally, the existence of S-PZQ was the primary reason that caused the extremely bitter and disgusting taste. ${ }^{16}$ A special program for the development of using RPZQ was set up by WHO. ${ }^{17}$ Single use of enantiopure PZQ against schistosomiasis would be valuable

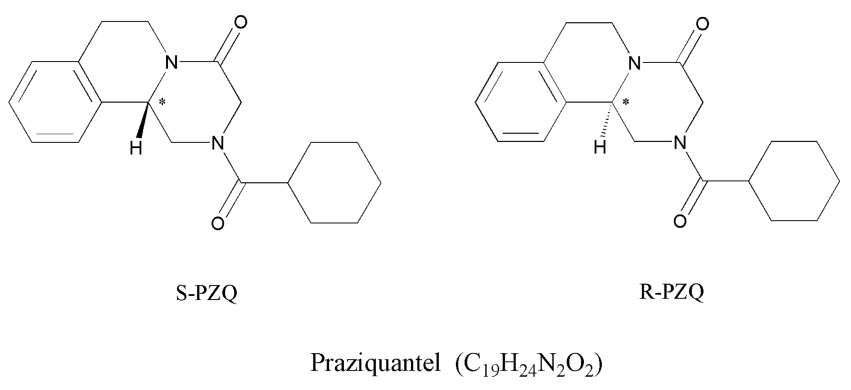

Fig. 1. The chemical structures of S-PZQ and R-PZQ. and promising. It would be necessary to establish a simple and useful method for determination of the enantiomeric composition of PZQ.

Many methods can be used to quantify the enantiomeric purity of a substance. Currently, highperformance liquid chromatography (HPLC) is the common method to determine the enantiomeric compositions of chiral compounds through the induction of chiral columns or derivatizing agents. ${ }^{18-21}$ Although HPLC can provide accurate result, it is complex, expensive and time-consuming. The ultraviolet (UV) spectroscopy is a simple and convenient method that has been successfully used to determine enantiomeric composition such as metalaxyl and ibuprofen. ${ }^{22}$ The UV absorption spectra of the different enantiomers of a racemate compound are identical in an achiral medium so that theoretically, the UV spectroscopy without chiral medium cannot determine the enantiomeric composition. With the addition of a chiral reagent (i.e., selector), the reagent and enantiomers can have diastereomeric interaction and then form enveloping substance, leading to a slight difference in their UV spectra, which can be employed to determine the enantiomeric composition through the quantitative model based on multivariate regression technique. Partial least squares (PLS), a very practical regression algorithm, is often used for establishing a quantitation model. ${ }^{23-27}$ Among chiral reagents used in chiral medium, cyclodextrins (CDs) are usually adopted but the price of CDs is expensive. Another most common chiral auxiliary is sucrose, which has been demonstrated to stereoselectively bind to chiral molecules to form complex and changeable conformations as well as produce diastereomeric interactions. ${ }^{28,29}$ Consequently, the determination of enantiomeric composition is also effective with a system based on sucrose by UV spectroscopy method.

In the present study, a simple and useful method to determine the enantiomeric composition of PZQ was proposed by UV spectroscopy combined with PLS regression model. The linear sugar sucrose was chosen to replace the expensive $\beta$-CD as the chiral reagent. ${ }^{30}$ The PLS quantitative model was established and optimized. Then the model was also applied to determine the enantiomeric composition of PZQ tablets, which was validated by comparing with the result from the HPLC. Similarly, this established model could have a practical application to determination of the other drug products such as PZQ tablets. 


\section{Experimental}

\subsection{Materials}

R-PZQ, S-PZQ and racemate PZQ were kindly provided by Prof. Dequn Sun. Ultrapure water was prepared through Milli-Q water purification system (Mosheim, France) and was used to prepare the sucrose solution. Methyl alcohol was purchased from Tian Jin Fuyu Fine Chemical Co. Ltd. to dissolve the PZQ. Sucrose was purchased from Sinopharm Chemical Reagent Co. Ltd. PZQ tablet was produced by Shenyang Hongqi Pharmaceutical Co. Ltd. (No. 1509071). UV spectrophotometer (UV-2401PC SHMADZU) was employed for measuring the spectra of sample solutions.

\section{2. $\quad$ Methods}

Preparation of sample solution: raw material usually has a very high purity so that the total chemical composition of the solution is relatively simple. A simple composition of solution could permit a calibration set containing a small number of samples to establish the mathematical model. Besides, a same total concentration of all the solutions of PZQ samples was maintained for eliminating the absorbance disturbance due to the inconsistent total concentration. The enantiomer ratio just varied to observe the variety of spectrum. According to Chieu D. Tran's work about the comparison of property between $\beta$-CD and sucrose as the chiral reagent for the analysis of chiral pharmaceutical products, the mole ratio of sucrose and the total PZQ as 10:1 would be better. ${ }^{30}$ When the total concentration of the PZQ is $0.4 \mathrm{mg} / \mathrm{mL}$, UV spectra of sample solutions are appropriate according to the optical properties of PZQ.

To prepare the same concentration of $\mathrm{PZQ}$ solution, stock solutions of $1.6 \mathrm{mg} / \mathrm{mL}$ were obtained by dissolving S-PZQ, R-PZQ and racemate PZQ in appropriate amount of methyl alcohol, respectively. The solution of sucrose $(5.8 \mathrm{mg} / \mathrm{mL})$ was prepared as the chiral medium. S-PZQ and R-PZQ were mixed with the racemate $P Z Q$, respectively, to make the solutions in which the proportions of S-PZQ varied from 1.00 to 0.20. All sample solutions were added with the same amount of sucrose solution (to keep the mole ratio 10:1), so that the total concentration of the PZQ in all sample solutions was same and just the ratios of S-PZQ varied.
The solution of the PZQ tablet was prepared as real sample for testing the quantitative model. The concentration of real sample is same as that of the PZQ solution. Six samples of PZQ tablets were prepared. Besides, the ratio of S-PZQ in tablet as the reference value was determined by using HPLC.

The spectra of the sample solutions were recorded with a UV-Vis spectrometer using a $1.0 \mathrm{~cm}$ path length quartz cell over the wavelength range from $190 \mathrm{~nm}$ to $500 \mathrm{~nm}$. The spectral bandwidth and data point interval are $1.0 \mathrm{~nm}$ and $0.2 \mathrm{~nm}$, respectively. The spectral data will be analyzed by TQ ANALYST 8 (Thermo SCIENTIFIC) to establish the PLS quantitative model. Full crossvalidation was used for determining the number of latent variables. The data of the PZQ tablets were used as an independent sample set to test the PLS model.

\section{Results and Discussion}

\subsection{The $U V$ spectrum}

UV absorption spectra of 33 solutions are shown in Fig. 2. There is a maximum of absorbance in the region from $220 \mathrm{~nm}$ to $230 \mathrm{~nm}$ even more than 2.8 which is the absorption of solvent, whereas the region from $260 \mathrm{~nm}$ to $270 \mathrm{~nm}$ has the authentic absorption peak whose value is about 0.5 corresponding to the characteristic absorption of PZQ. The inset of Fig. 2 shows a variational trend with different enantiomeric compositions, especially at the region near the peak or the valley that coincides with related spectroscopic studies on the other chiral compounds. ${ }^{22,31}$ The mole fraction of the S-PZQ varied from 0.20 to 1.00 in the 33 sample solutions. Each fraction value was corresponding to each spectrum, respectively. These spectra display a whole falling trend with the decrease of the ratio of S-PZQ.

\subsection{Sample division and spectral pretreatment}

To establish a model for calibration, samples were divided into two subsets. Twenty seven samples were chosen as the calibration set and six samples were chosen as the validation set based on Kennard-Stone (KS) algorithm. Different pretreatments were applied to the spectral data, including standard normal variate (SNV), Savitzky-Golay 


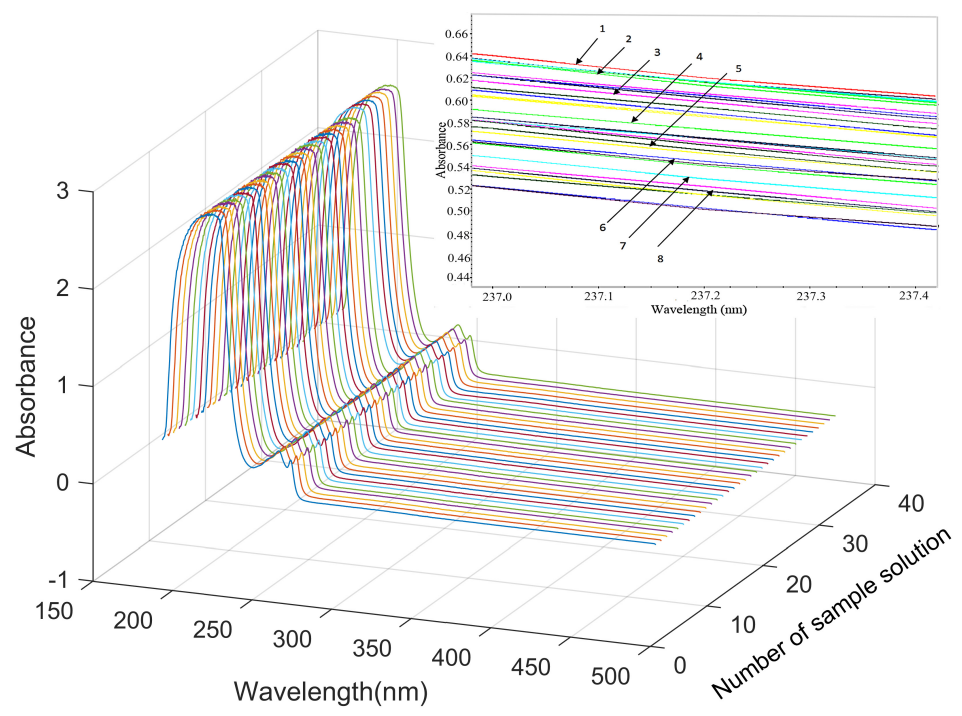

Fig. 2. The whole UV spectra of 33 solutions of PZQ from $190 \mathrm{~nm}$ to $500 \mathrm{~nm}$. The region was selected for analysis from $287.2 \mathrm{~nm}$ to $236.6 \mathrm{~nm}$, where the maximum of absorbance was about 0.5. Inset: Expanded spectra were from $237.4 \mathrm{~nm}$ to $237.0 \mathrm{~nm}$. The spectral bands pointed with arrows and number corresponding to the different ratios of S-PZQ enantiomer (the ratio from 1 to 8 corresponding to $1.00,0.96,0.92,0.80,0.72,0.68,0.62$ and 0.58 , respectively).

smoothing (SG), first derivation (1st D) and second derivation (2nd D). Selection of appropriate wavelength region was also involved. Generally, SNV can process samples with different effective path lengths when an independent measure of sample path length is very difficult or impossible. It is often applied in diffuse reflectance near infrared spectrum of solid sample. The SG smoothing filter is very useful for improving the appearance of peaks obscured by random noise. If the SG smoothing filter was selected, the settings for data points and polynomial order determined the degree of smoothing. It can be applied to the original spectrum or to the first or second derivative spectrum. The pretreatment results are shown in Table 1 . Because the higher the value of correlation coefficient $(r)$, the smaller the RMSEC and the RMSEP, the better performance of the PLS model was. So, the best result of direct computation with raw data after SG smoothing was found in comparison with the other methods. Usually, the data would have better results if some pretreatment approaches such as smooth, derivation and so on were used. As shown in Table 1, when the raw data were smoothed with SG, the linear correlation is better than raw data. But unsatisfactory results were found if first or second derivative was used. Besides, for the present samples preprocessed by SNV, no optimal results were obtained. Therefore, the optimal result was achieved by using original data with SG smoothing.
On the contrary, the selection of the appropriate spectral region is also important to get an optimal result. Though the region should be located at the spectral part where sample's spectra could have an obvious absorption that varied with sample's concentrations, it is not easy to find a certain wavelength region for attaining a good linear relationship. First, the recommended wavelength interval $(271.6-263.4 \mathrm{~nm})$ from the analysis software (TQ) was used, but the result was not satisfied. Then, the full-wavelength band was considered and the result had a certain improvement but not the best. Through many attempts based on the recommended wavelength interval, the optimal spectral region from $287.2 \mathrm{~nm}$ to $236.6 \mathrm{~nm}$ was selected for the PLS model (shown in Fig. 2).

\subsection{Establishment of PLS model}

Before the PLS model was established, outlier samples were tested. Figure 3(a) shows that no abnormal sample is found. The results of PLS analysis are shown in Fig. 3(b). The optimal PLS model used five latent variables which could indicate R-PZQ and S-PZQ as well as sucrose, methyl alcohol and water. The correlation coefficients of calibration set and validation set were 0.9971 and 0.9972, respectively. The RMSEC and the RMSEP values were 0.0167 and 0.0129 , respectively. The mean and standard deviation (SD) of the mole 
Table 1. The results of pretreatment of the spectral data by some methods.

\begin{tabular}{|c|c|c|c|c|c|}
\hline \multirow[b]{2}{*}{ Pretreatment } & \multirow{2}{*}{$\begin{array}{l}\text { Wavelength } \\
\qquad\left(\mathrm{nm}^{-1}\right)\end{array}$} & \multicolumn{2}{|c|}{ Calibration set } & \multicolumn{2}{|c|}{ Validation set } \\
\hline & & $r^{\mathrm{a}}$ & RMSEC & $R$ & RMSEP \\
\hline \multirow[t]{2}{*}{$\mathrm{SNV}^{\mathrm{b}}$} & $263.4-271.6$ & 0.9839 & 0.0394 & 0.9934 & 0.0202 \\
\hline & 200-300 & 0.9989 & 0.0104 & 0.9774 & 0.0357 \\
\hline \multirow[t]{2}{*}{$\mathrm{SNV}, \mathrm{SG}(7,3)^{\mathrm{c}}$} & $263.4-271.6$ & 0.9813 & 0.0425 & 0.9897 & 0.0217 \\
\hline & 200-300 & 0.9990 & 0.0098 & 0.9839 & 0.0287 \\
\hline \multirow[t]{3}{*}{ Original $^{\mathrm{d}}$} & $263.4-271.6$ & 0.9839 & 0.0395 & 0.9888 & 0.0510 \\
\hline & $236.6-287.2$ & 0.9973 & 0.0164 & 0.9967 & 0.0150 \\
\hline & 190-500 & 0.9941 & 0.0241 & 0.9961 & 0.0191 \\
\hline Original, $\mathrm{SG}(7,3)$ & $236.6-287.2$ & 0.9971 & 0.0167 & 0.9972 & 0.0129 \\
\hline Original, $\mathrm{SG}(7,3)$ & $190-500$ & 0.9939 & 0.0245 & 0.9957 & 0.0200 \\
\hline Original, $\mathrm{SG}(9,5)$ & $236.6-287.2$ & 0.9969 & 0.0174 & 0.9966 & 0.0145 \\
\hline \multirow[t]{2}{*}{1 st $\mathrm{D}^{\mathrm{e}}$} & $263.4-271.6$ & 0.9825 & 0.0411 & 0.8966 & 0.1220 \\
\hline & $236.6-287.2$ & 0.9967 & 0.0180 & 0.9783 & 0.0353 \\
\hline \multirow[t]{2}{*}{ 1st D,SG(7,3) } & $263.4-271.6$ & 0.9828 & 0.0407 & 0.8685 & 0.1230 \\
\hline & $238-285.8$ & 0.9966 & 0.0180 & 0.9836 & 0.0291 \\
\hline 1st D,SG $(5,5)$ & $238-285.8$ & 0.9965 & 0.0184 & 0.9775 & 0.0443 \\
\hline \multirow[t]{2}{*}{$2 \mathrm{nd} \mathrm{D}^{\mathrm{f}}$} & $236.6-287.2$ & 0.9993 & 0.0081 & 0.5437 & 0.1220 \\
\hline & $190-500$ & 0.9839 & 0.0398 & 0.2845 & 0.1470 \\
\hline \multirow[t]{2}{*}{ 2nd D,SG $(7,3)$} & $236.6-287.2$ & 0.9940 & 0.0241 & 0.8644 & 0.0760 \\
\hline & 190-500 & 0.9472 & 0.0714 & 0.8623 & 0.1090 \\
\hline
\end{tabular}

${ }^{a}$ Correlation coefficient.

bStandard normal variate.

${ }^{\mathrm{c}}$ Savitzky-Golay with a 7-points window and a third-order polynomial.

${ }^{\mathrm{d}}$ The original spectral data which no pretreatments used.

eFirst derivation.

fecond derivation.

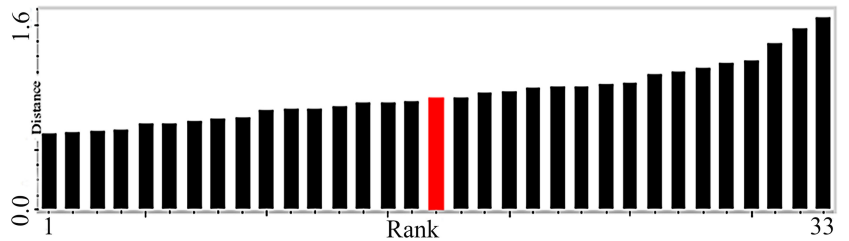

(a)

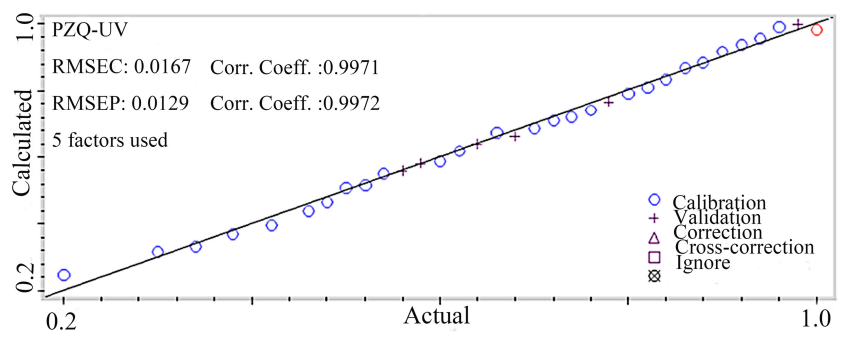

(b)

Fig. 3. (a) The result showed that no outliers were found. (b) The result of PLS analysis including the correlation coefficient of calibration set and validation set: 0.9971 and 0.9972 . The RMSEC and the RMSEP values were 0.0167 and 0.0129 , respectively. Five factors were used for the model.
Table 2. The parameters about the ratios in the model.

\begin{tabular}{lccccccc}
\hline & \multicolumn{3}{c}{ Calibration set } & & \multicolumn{3}{c}{ Validation set } \\
\cline { 2 - 3 } \cline { 5 - 7 } $\begin{array}{l}\text { Quality } \\
\text { indicator }\end{array}$ & Range & Mean & SD $^{\mathrm{a}}$ & & Range & Mean & SD \\
\hline $\begin{array}{c}\text { Ratio of } \\
\text { S-PZQ }\end{array}$ & $0.200-1.00$ & 0.661 & 0.220 & $0.560-0.980$ & 0.703 & 0.161 \\
& & & & & & & \\
\hline
\end{tabular}

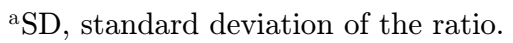

ratio of the calibration set were 0.661 and 0.220 and those of the validation set were 0.703 and 0.161 , respectively (shown in Table 2). Figure 4 shows that the calculated values are very close to the actual values, and the absolute value of error is not more than 0.05 . The average error is 0.02 .

\subsection{Test of the model by real samples}

First, the ratio of S-PZQ in tablets was measured by using HPLC as the reference method. The result of S-PZQ ratio value was 0.492 . The spectra of the six 


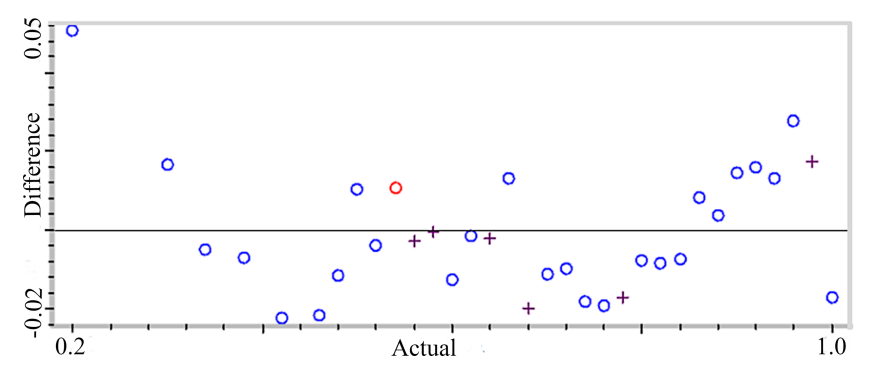

Fig. 4. This plot shows the differences between the calculated values relative to the actual values and the absolute value not more than 0.05 and the average value was 0.02 , which suggested the accuracy of the model.

solutions of PZQ tablets were almost same as those of racemic PZQ shown in Fig. 5. The six spectra were put into PLS model to predict their enantiomeric compositions. When all the six spectra were used as the test set, the calculated results and their related errors with reference value were relatively high and unacceptable and the correlation coefficient of the validation set was 0.9905. However, when a random spectrum among the six spectra was chosen as a real calibration sample to add to the calibration set, and the remaining spectra as the test set, the calculated results were good and the correlation coefficient of validation set was improved (0.9960). The predicted ratios of S-PZQ for the remaining five samples were $0.474,0.510$, $0.506,0.507$ and 0.498 , respectively. Their related error compared with the reference value was from $-3.66 \%$ to $3.66 \%$, indicating a good prediction ability of the PLS model for real samples of PZQ tablet (shown in Tables 3(a) and 3(b)). The possible reason for the difference between the two ways used for testing the model would be that the initial model did not include the information of the tablet excipients. If the information of the excipients has little effect on the PLS model only including chemical raw materials, the predicted results for real samples might be satisfactory. Actually, preparation of samples that is very similar or identical to real samples for determination of real sample composition would be an ideal approach. However, this approach was unrealistic because the prescription of excipients of PZQ tablets could not be available. Therefore, for practical application, a convenient strategy for this issue is to introduce real sample into calibration set, so that the information on excipients of real sample can be involved. Our results demonstrated that the introduction of only a real sample into calibration set could significantly improve the predicted capability of PLS model (Tables 3(a) and 3(b)). The range of related errors had a marked reduction. The results of test of PLS model suggested that it would be essential to consider the effect of excipients to get better prediction results if the real tablet was used as the independent external validation.

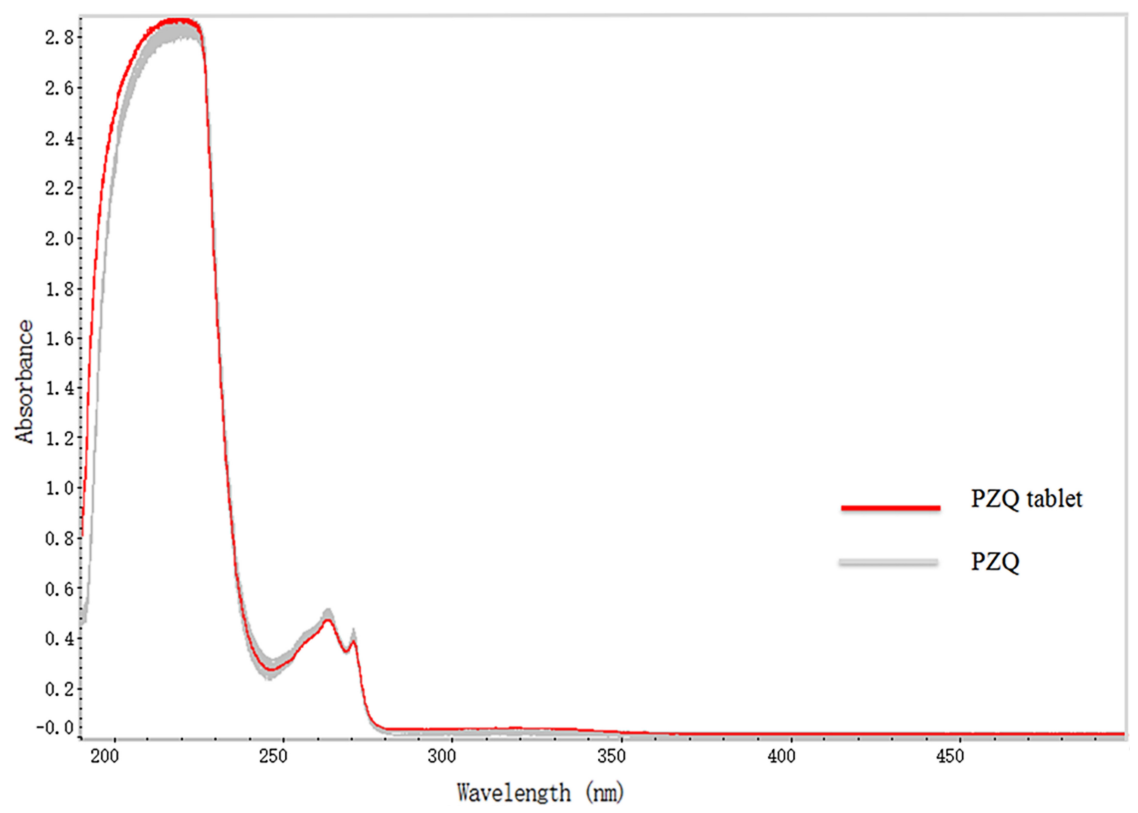

Fig. 5. The spectra of the six solutions of PZQ tablets. It had no abnormal phenomenon compared with the model. 
Table 3(a). The results of no sample of tablet involved in the calibration set.

\begin{tabular}{lccc}
\hline Samples & $\begin{array}{c}\text { Reference } \\
\text { value }\end{array}$ & $\begin{array}{c}\text { Calculated } \\
\text { value }\end{array}$ & $\begin{array}{c}\text { Related } \\
\text { error (\%) }\end{array}$ \\
\hline 1 & 0.492 & 0.497 & 1.02 \\
2 & & 0.538 & 9.35 \\
3 & & 0.532 & 8.13 \\
4 & & 0.528 & 7.32 \\
5 & & 0.517 & 5.08 \\
6 & & 0.522 & 6.10 \\
Average & & 0.522 & 6.10 \\
\hline
\end{tabular}

Table 3(b). The results of a random sample of tablet selected to add to the calibration set.

\begin{tabular}{lccc}
\hline Samples & $\begin{array}{c}\text { Reference } \\
\text { value }\end{array}$ & $\begin{array}{c}\text { Calculated } \\
\text { value }\end{array}$ & $\begin{array}{c}\text { Related } \\
\text { error (\%) }\end{array}$ \\
\hline 1 & 0.492 & 0.474 & -3.66 \\
2 & & 0.510 & 3.66 \\
3 & & 0.506 & 2.84 \\
4 & & 0.503 & 2.24 \\
6 & & 0.497 & 1.02 \\
Average & & 0.498 & 1.22 \\
\hline
\end{tabular}

\section{Conclusions}

In this study, small spectral variations in the UV spectra of PZQ solutions mixed with sucrose were observed when the enantiomeric composition varies by keeping the total concentration of PZQ constant at the same time. Sucrose is efficient in inducing diastereomeric interactions with $\mathrm{R}$ - and S-enantiomer, which result in accurate determination of enantiomeric compositions.

A simple and useful quantitative model was established for the determination of enantiomeric composition of PZQ with the UV spectroscopy combined with the PLS analysis. As the results show, the correlation coefficients of calibration set and validation set were 0.9971 and 0.9972 , respectively. The RMSEC and RMSEP values were 0.0167 and 0.0129 , respectively. Hence, the model has a satisfied performance and accuracy. When the PZQ tablet data were predicted by the PLS model, the related errors of the ratios of S-PZQ between the calculated values through the model and the reference values were less than $4 \%$, indicating that a good predicted ability was achieved to quantify the enantiomeric composition of the real finished products (i.e., PZQ tablets).

In summary, the method used here could be feasible and practical. Moreover, the established method could be a useful approach potentially applied to control the productive process as well as to be a convenient screening of the finished products for their quality control.

\section{Conflict of interest}

The authors have declared no conflict of interest.

\section{Acknowledgments}

This work was supported by National Natural Science Foundation of China (No. 81102504), Key Technologies R\&D Program of Shandong Province (No. 2016GSF201003) and the Scientific Research Foundation for the Returned Overseas Chinese Scholars, State Education Ministry (the 50th Batch).

\section{References}

1. D.-E. Drayer, "Pharmacodynamic and pharmacokinetic differences between drug enantiomers in humans: An overview," Clin. Pharmacol. Ther. 40, 125-133 (1986).

2. B. Waldeck, "Biological significance of the enantiomeric purity of drugs," Chirality 5, 350-355 (1993).

3. W.-H. De Camp, "Chiral drugs: The FDA perspective on manufacturing and control," J. Pharmaceut. Biomed. Anal. 11, 1167-1172 (1993).

4. L. Rigoni, S. Venti, M. Bevilacqua, R. Bucci, A.-D. Magrì, A.-L. Magrì, F. Marini, "Quantification of the enantiomeric excess of two APIs by means of near infrared spectroscopy and chemometrics," Chemometr. Intell. Lab. 133, 149-156 (2014).

5. P. Lu, L. You, H.-Y. Hua, "Clinical observation on the efficacies of praziquantel for liver fibrosis in patients with advanced schistosomiasis," J. Trop. Dis. Parasitol. 4, 204-207 (2015).

6. Y. Yan, J.-M. Wang, Y. Jiang, X.-H. Li, Y. Liu, C.-G. Zhu, Y.-J. Shi, "Experiment of praziquantel rectal administration in treatment of schistosomiasis in mice," Chin. J. Schisto Control 6, 674-676 (2011).

7. W.-L. Wu, X.-Z. Zhou, Y.-Y. Huang, "Longterm efficacy of pyquiton on Schistosomiasis japonica," Chin. J. Infect. Dis. 4, 21-24 (2004).

8. Z.-J. Wen, "Therapeutic effect observation of schistosomiasis chronic diarrhea patients are treated by spraying praziquantel solution under the colonoscope and oral praziquantel tablet," J. Pathog. Biol. 11, 1031-1032 (2013).

9. WHO, "Prevention and control of schistosomiasis and soil-transmitted helminthiasis," World Health Organization Technical Report 912, i (2002). 
10. M. Tanaka, H. Ohmae, H. Utsunomiya, T. Nara, Y. Irie, K. Yasuraoka, "A comparison of the antischistosomal effect of levo- and dextro-praziquantel on Schistosoma japonicum and S. mansoni in mice," Am. J. Trop. Med. Hyg. 41, 198-203 (1989).

11. Y.-H. Liu, M.-X. Qian, X.-G. Wang, J. Jia, Q.-N. Wang, Y.-F. Jiang, R.-Q. Wang, S.-H. Yan, B.-Y. Chen, J.-S. Li, "Comparative efficacy of praziquantel and its optic isomers in experimental therapy of Schistosomiasis japonica in rabbits," Chin. Med. J. 99, 935 (1986).

12. S.-H. Xiao, B.-A. Catto, "Comparative in vitro and in vivo activity of racemic praziquantel and its levorotated isomer on Schistosoma mansoni," J. Infect. Dis. 159, 589-592 (1989).

13. Y. Zheng, L. Dong, C. Hu, B. Zhao, C. Yang, C. Xia and D. Sun, "Development of chiral praziquantel analogues as potential drug candidates with activity to juvenile Schistosoma japonicum," Bioorg. Med. Chem. Lett. 24, 4223 (2014).

14. S.-H. Xiao, J.-Q. You, J.-Y. Mei, Y.-Q. Hu, D.-H. Zhou, B.-A. Catto, "In vitro and in vivo effect of levopraziquantel, dextropraziquantel versus racemic praziquantel on different developmental stages of Schistosoma japonicum," Chin. J. Parasitol. Parasit. Dis. 16, 335 (1998).

15. D. Cioli, S.-S. Botros, K. Wheatcroft-Francklow, A. Mbaye, V. Southgate, L.-A. Tchuente, L. Pica-Mattoccia, A.-R. Troiani, S.-H. El-Din, A.-N. Sabra, J. Albin, D. Engels, M.-J. Doenhoff, "Determination of ED50 values for praziquantel in praziquantel-resistant and -susceptible Schistosoma mansoni isolates," Int. J. Parasitol. 34, 979-987 (2004).

16. P. Olliaro, P. Delgado-Romero and J. Keiser, "The little we know about the pharmacokinetics and pharmacodynamics of praziquantel (racemate and R-enantiomer)," J. Antimicrob. Chemoth. 69, 863870 (2014).

17. H.-N. Wang, Z.-Z. Fang, Y. Zheng, K. Zhou, C.-Y. Hu, K.-W. Krausz, D.-Q. Sun, J.-R. Idle, F.-J. Gonzalez, "Metabolic profiling of praziquantel enantiomers," Biochem. Pharmacol. 90, 166-178 (2014).

18. M. Yang, W.-Y. Zhong, W. Hou, "Advances in research on analytical methods of chiral drugs," Prog. Pharm. Sci. 38, 209-214 (2014).

19. L.-X. Lin, X.-H. Xue, M.-F. Fan, "Analysis of pyquiton by reversed phase high-performance liquid chromatography," Acta Pharmaceut. Sin. 3, 224228 (1985).

20. X.-H. Zheng, Q. Shao, H.-Y. Jing, "Enantiomers detection of praziquantel intermediates by RPHPLC after GITC pre-column derivatization," WORLD SCI-TECH RED 3, 626-629 (2016).
21. Q.-Z. Wu, R. Chen, S.-L. Qiao, "Enantiospecific analysis of pregabalin by HPLC with chiral precolumn derivatization," J. China Pharm. Univ. 6, 523-526 (2007).

22. Q.-Q. Li, L.-J. Wu, W. Liu, J.-L. Cao, X. Duan, Y. Huang, S.-G. Min, "UV spectroscopy coupled with partial least squares to determine the enantiomeric composition in chiral drugs," Spectrosc. Spect. Anal. 2, 500-504 (2011).

23. D.-C. Gu, M.-J. Zou, X.-X. Guo, P. Yu, Z.-W. Lin, T. Hu, Y.-F. Wu, Y. Liu, J.-H. Gan, S.-Q. Sun, X.-C. Wang, C.-H. Xu, "A rapid analytical and quantitative evaluation of formaldehyde in squid based on Tri-step IR and partial least squares (PLS)," Food Chem. 229, 458-463 (2017).

24. A. Palou, A. Miró, M. Blanco, R. Larraz, J.-F. Gómez, T. Martínez, J.-M. González and M. Alcalà, "Calibration sets selection strategy for the construction of robust PLS models for prediction of biodiesel/diesel blends physico-chemical properties using NIR spectroscopy," Spectrochim. Acta A: Mol. Biomol. Spectrosc. 180, 119-126 (2017).

25. Y.-Z. Liang, R.-Q. Yu, Y.-L. Xie, "Partial leastsquares regression applied to pharmaceutical analysis," Anal. Chem. 7, 588-592 (1989).

26. L.-M. De Souza, F.-B. De Santana, L.-C. Gontijo, S.-J. Mazivila, N.-W. Borges, "Quantification of adulterations in extra virgin flaxseed oil using MIR and PLS," Food Chem. 182, 35-40 (2015).

27. J.-B. Liu, Y. Ren, P.-X. Zhang, "Spectrophotometric determination of pharmaceutical compositions by partial least squares method and principal component regression method," Anal. Chem. 10, 887-892 (1990).

28. K.-W. Busch, I.-M. Swamidoss, S.-O. Fakayode, M.A. Busc, "Determination of the enantiomeric composition of guest molecules by chemometric analysis of the UV-visible spectra of cyclodextrin guest-host complexes," J. Am. Chem. Soc. 125, 1690-1691 (2003).

29. Q.-Q. Li, Y. Huag, J. Duan, L.-J. Wu, G. Tang, Y.W. Zhu and S.-G. Min, "Sucrose as chiral selector for determining enantiomeric composition of metalaxyl by UV-vis spectroscopy and PLS regression," Spectrochim. Acta A: Mol. Biomol. Spectrosc. 101, 349-355 (2013).

30. C.-D. Tran, D. Oliveira, V.-I. Grishko, "Determination of enantiomeric compositions of amino acids by nearinfrared spectrometry through complexation with carbohydrate," Anal. Chem. 75, 6455-6462 (2003).

31. S.-O. Fakayode, I.-M. Swamidoss, M.-A. Busch, K.W. Busch, "Determination of the enantiomeric composition of some molecules of pharmaceutical interest by chemometric analysis of the UV spectra of guest-host complexes formed with modified cyclodextrins," Talanta 65, 838-845 (2005). 